\title{
Inverse structure functions of temperature in grid-generated turbulence
}

Cite as: Physics of Fluids 16, 2126 (2004); https://doi.org/10.1063/1.1710890

Submitted: 03 October 2003 . Accepted: 27 February 2004 . Published Online: 04 May 2004

\section{S. Beaulac, and L. Mydlarski}

\section{ARTICLES YOU MAY BE INTERESTED IN}

Inverse structure functions in the canonical wind turbine array boundary layer

Journal of Renewable and Sustainable Energy 8, 053310 (2016); https://

doi.org/10.1063/1.4966228

Simultaneous velocity-temperature measurements in the heated wake of a cylinder with implications for the modeling of turbulent passive scalars

Physics of Fluids 23, 055107 (2011); https://doi.org/10.1063/1.3586802

Dependence on the initial conditions of scalar mixing in the turbulent wake of a circular

cylinder

Physics of Fluids 16, 3161 (2004); https://doi.org/10.1063/1.1766033

\section{Physics of Fluids}




\title{
Inverse structure functions of temperature in grid-generated turbulence
}

\author{
S. Beaulac and L. Mydlarski ${ }^{\text {a) }}$ \\ Department of Mechanical Engineering, McGill University, 817 Sherbrooke St. W., Montréal, \\ Québec H3A 2K6, Canada
}

(Received 3 October 2003; accepted 27 February 2004; published online 4 May 2004)

\begin{abstract}
Jensen [Phys. Rev. Lett. 83, 76 (1999)] proposed a new technique to study the scaling behavior of turbulent velocity fields. Inverse structure functions - defined as average moments of distances (or times) corresponding to a specified difference of a turbulent quantity-were used to investigate the intermittency of the turbulent velocity field. The present Brief Communication employs inverse structure functions to study the behavior of a passive scalar (temperature) in high-Reynolds-number grid-generated turbulence. It is shown that the scaling exponents of inverse structure functions of temperature are significantly different than those of the longitudinal and transverse velocity. Such a result is attributed to the higher level of intermittency associated with passive scalar fields. (C) 2004 American Institute of Physics. [DOI: 10.1063/1.1710890]
\end{abstract}

Since Kolmogorov's 1941 prediction $^{1,2}$ regarding the small-scale structure of a turbulent velocity field, structure functions have been commonly used in the study of turbulence. They are defined as statistical moments of velocity differences over a specified scale:

$$
\left\langle\left(\Delta u_{i}\left(r_{j}\right)\right)^{n}\right\rangle \equiv\left\langle\left(u_{i}\left(x_{j}+r_{j}\right)-u_{i}\left(x_{j}\right)\right)^{n}\right\rangle,
$$

where $u_{i}$ is a turbulent velocity fluctuation and $r_{j}$ is a separation (often measured in the direction of the mean flow and calculated using Taylor's hypothesis). Kolmogorov ${ }^{1,2}$ predicted the inertial-range scaling behavior of the $n$ th-order structure function to be

$$
\left\langle\left(\Delta u_{i}(r)\right)^{n}\right\rangle=f(\epsilon, r) \propto r^{\zeta_{n}},
$$

where, from dimensional considerations,

$$
\zeta_{n}=n / 3 \text {. }
$$

Note that $r=\left|r_{j}\right|$ and $\epsilon$ is the dissipation rate of turbulent kinetic energy (which, in the inertial subrange at large Reynolds numbers, is equal to the spectral energy transfer rate).

Since 1941, it has become apparent that the scaling implied by Eq. (3) needs refining. ${ }^{3-8}$ Due to the large variations in space and time of $\epsilon$ (called internal intermittency), the probability density functions (PDFs) of $\Delta u_{i}(r)$ cannot be collapsed by a rescaling scheme and the dependence of $\zeta_{n}$ on $n$ becomes nonlinear. The relationship between $\zeta_{n}$ and $n$ has been the focus of much research-see Ref. 8 for a review.

To consider the scaling and intermittency of turbulent velocity fields from a novel point of view, Jensen ${ }^{9}$ proposed inverting structure functions and studying average moments of distances between two points possessing a specified velocity difference. Whereas traditional structure functions quantify statistical moments of velocity differences over a specified scale, inverse structure functions ${ }^{10}$ are defined to be average moments of distances (or times) corresponding to a specified difference of a turbulent quantity. Using velocity as

a)Electronic mail: laurent.mydlarski@mcgill.ca an example, they can be written as $\left\langle\left(r\left(\left|\Delta u_{i}\right|\right)\right)^{n}\right\rangle$ when considering spatial separations or $\left\langle\left(t\left(\left|\Delta u_{i}\right|\right)\right)^{n}\right\rangle$ when considering temporal separations. $r\left(\left|\Delta u_{i}\right|\right)$ or $t\left(\left|\Delta u_{i}\right|\right)$ represent the minimal separation in space or time, respectively, for which the magnitude of the measured velocity difference is $\left|\Delta u_{i}\right|$. The averaging is performed in the same manner as for (traditional) structure functions (i.e., over space or time for experiments such as those described herein).

Just as an inertial-range scaling behavior of regular structure functions is expected, so would be that of inverse structure functions, i.e.,

$$
\left\langle\left(r\left(\left|\Delta u_{i}\right|\right)\right)^{n}\right\rangle \propto\left|\Delta u_{i}\right|^{\delta_{n}} .
$$

However, the relationship between $\zeta_{n}$ and $\delta_{n}$ is non trivial. Using GOY shell model computations, ${ }^{11-13}$ Jensen $^{9}$ calculated inverse structure functions up to order 8 and showed that i) strong intermittency effects are also observed for $\delta_{n}$ (i.e., $\left.\delta_{n} \neq 3 n\right)$, ii) $\delta_{n} \neq 1 / \zeta_{n}$ and iii) the PDFs of $r$ for a given $\Delta u_{i}$ are non-Gaussian for both small and large scales.

Using experimental and synthetic data, inverse structure functions of the velocity field have also been used to investigate the intermediate dissipation range. ${ }^{14}$ It was shown that the latter is more extended than when studied by means of traditional structure functions. ${ }^{15-17}$ Inverse structure functions have also been used to study two-dimensional turbulence. ${ }^{18,19}$

The purpose of the present Brief Communication is to investigate the scaling and intermittency of a turbulent passive scalar field by means of inverse structure functions. In doing so, we also present experimental measurements of inverse structure functions of the longitudinal and transverse velocity fields and compare them with those of the scalar.

The measurements were made in the $0.91 \mathrm{~m} \times 0.91$ $\mathrm{m} \times 9.1 \mathrm{~m}$ low-speed, low-background-turbulence wind tunnel in the Sibley School of Mechanical and Aerospace Engineering at Cornell University. The flow is homogeneous, quasi-isotropic, high-Reynolds-number, grid-generated turbulence. Large Reynolds (and Péclet) numbers were obtained 
TABLE I. Flow parameters. All measurements were made at $x / M=62$. The $R_{\lambda}=140$ case used the active grid operating in its synchronous mode. The other two cases employed its random mode. (See Refs. 21 and 23.)

\begin{tabular}{llll}
\hline \hline$R_{\lambda}$ & 140 & 306 & 582 \\
\hline$\langle U\rangle(\mathrm{m} / \mathrm{s})$ & 3.3 & 3.3 & 7.0 \\
$d T / d y(\mathrm{~K} / \mathrm{m})$ & 2.5 & 2.7 & 3.6 \\
$\left\langle u^{2}\right\rangle\left(\mathrm{m}^{2} / \mathrm{s}^{2}\right)$ & 0.0290 & 0.0911 & 0.583 \\
$\left\langle v^{2}\right\rangle\left(\mathrm{m}^{2} / \mathrm{s}^{2}\right)$ & 0.0209 & 0.0594 & 0.424 \\
$\left\langle\theta^{2}\right\rangle\left(\mathrm{K}^{2}\right)$ & 0.176 & 0.800 & 1.07 \\
$\epsilon\left(\mathrm{m}^{2} / \mathrm{s}^{3}\right)$ & 0.0418 & 0.0833 & 0.940 \\
$\epsilon_{\theta}\left(\mathrm{K}^{2} / \mathrm{s}\right)$ & 0.277 & 0.799 & 1.74 \\
$\eta\left(=\left(\nu^{3} / \epsilon\right)^{1 / 4}\right)(\mathrm{mm})$ & 0.55 & 0.47 & 0.26 \\
$\tau\left(=(\nu / \epsilon)^{1 / 2}\right)(\mathrm{s})$ & 0.0193 & 0.0139 & 0.00413 \\
\hline \hline
\end{tabular}

by use of an active grid. ${ }^{20-22}$ Passive scalar (temperature) fluctuations were produced by the action of the turbulent velocity field against an imposed mean temperature gradient. The latter was generated by differentially heating parallel ribbons located at the entrance to the wind tunnel plenum chamber. The characteristics of the velocity and thermal fields, as well as the details of the apparatus, are documented in Refs. 21 and 23. Hot-wire anemometry and cold-wire thermometry were employed to measure the respective fields. The hot-wire signals were compensated for the temperature
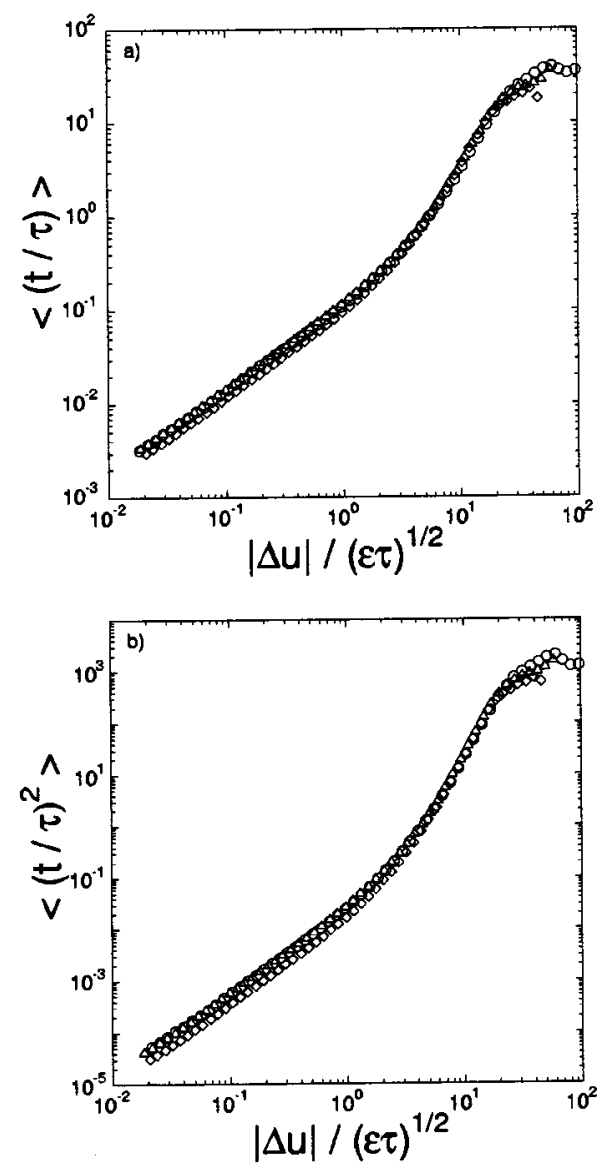

FIG. 1. Inverse structure functions of $u$ of the (a) first order and (b) second order. $R_{\lambda}=140(\diamond), R_{\lambda}=306(\triangle), R_{\lambda}=582(\bigcirc)$.
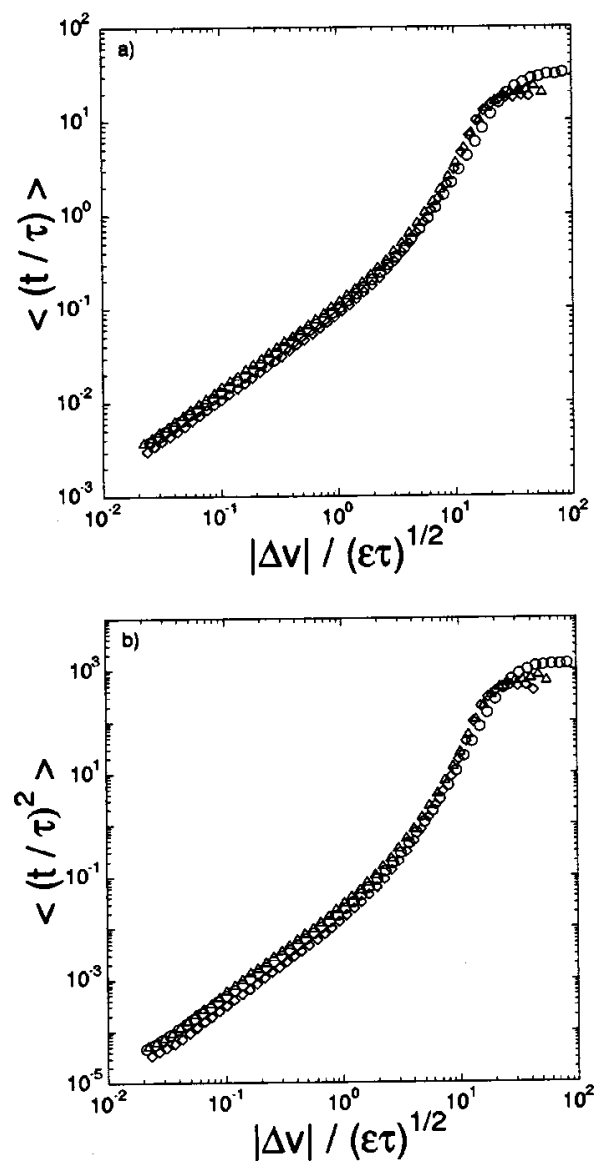

FIG. 2. Inverse structure functions of $v$ of the (a) first order and (b) second order. $R_{\lambda}=140(\diamond), R_{\lambda}=306(\triangle), R_{\lambda}=582(\bigcirc)$.

fluctuations by means of a modified King's law with temperature-dependent coefficients. ${ }^{24}$

Measurements are presented for three Reynolds numbers: $R_{\lambda}=140,306$, and 582, where $R_{\lambda}=\left\langle u^{2}\right\rangle[15 /(\nu \epsilon)]^{1 / 2}$. The dissipation rate of turbulent kinetic energy is estimated from $\epsilon=15 \nu \int_{0}^{\infty} k_{1}^{2} F_{11}\left(k_{1}\right) d k_{1}$, where $F_{11}\left(k_{1}\right)$ is the onedimensional, longitudinal power spectrum of $u$. The flow parameters for these three cases are summarized in Table I. More details can be found in Ref. 23.

Figures 1 and 2 plot the first- and second-order temporal inverse structure functions of $u$ and $v$ for the three Reynolds numbers. ${ }^{25}$ Both behave in very similar fashions. (Spatial inverse structure functions can be obtained from the present results by multiplying the ordinates of the figures by $(\tau\langle U\rangle / \eta)^{n}$, where $n$ is the order of the inverse structure function under consideration.) At small scales, the first- and second-order inverse structure functions exhibit slopes of approximately 1 and 2, respectively, as required by the smallscale, "laminar," asymptotic limit of $\Delta u^{n} \propto r^{n}$. At intermediate separations, another scaling range is observed. The slopes of these ranges are given in Table II. We observe the magnitudes of the inverse structure function scaling exponents for $R_{\lambda}=140$ to be slightly higher than those at the other two Reynolds numbers. For these highest Reynolds numbers, the values of $\delta_{1}^{u}$ and $\delta_{1}^{v}$ (approximately 2.0), $\delta_{2}^{u}$ and $\delta_{2}^{v}$ (approximately 3.9) and those of $\delta_{3}^{u}$ and $\delta_{3}^{v}$ (approximately 5.6) are in 
TABLE II. The scaling exponents of the traditional and inverse structure functions of $u, v$, and $\theta$ for orders 1,2 , and 3 . The exponents are determined by fitting a best fit power-law to the scaling range. The odd-ordered traditional structure functions are calculated using averages of moments of the absolute value of the differences.

\begin{tabular}{llllc}
\hline \hline$R_{\lambda}$ & 140 & 306 & 582 & Jensen (Ref. 9) \\
\hline$\zeta_{1}^{u}$ & 0.29 & 0.33 & 0.32 & 0.39 \\
$\zeta_{1}^{v}$ & 0.27 & 0.30 & 0.34 & $\ldots$ \\
$\zeta_{1}^{\theta}$ & 0.36 & 0.36 & 0.37 & $\ldots$ \\
$\zeta_{2}^{u}$ & 0.52 & 0.63 & 0.62 & 0.73 \\
$\zeta_{2}^{v}$ & 0.48 & 0.58 & 0.63 & $\ldots$ \\
$\zeta_{2}^{\theta}$ & 0.58 & 0.61 & 0.62 & $\ldots$ \\
$\zeta_{3}^{u}$ & 0.80 & 0.90 & 0.89 & 1.0 \\
$\zeta_{3}^{v}$ & 0.65 & 0.84 & 0.90 & $\ldots$ \\
$\zeta_{3}^{\theta}$ & 0.74 & 0.79 & 0.81 & $\ldots$ \\
$\delta_{1}^{u}$ & 2.1 & 2.0 & 2.0 & 2.04 \\
$\delta_{1}^{v}$ & 2.5 & 2.1 & 2.1 & $\ldots$ \\
$\delta_{1}^{\theta}$ & 1.7 & 1.6 & 1.5 & $\ldots$ \\
$\delta_{2}^{u}$ & 4.1 & 3.9 & 3.9 & 3.70 \\
$\delta_{2}^{v}$ & 4.6 & 4.0 & 3.9 & $\ldots$ \\
$\delta_{2}^{\theta}$ & 3.1 & 2.8 & 2.7 & $\ldots$ \\
$\delta_{3}^{u}$ & 5.8 & 5.6 & 5.6 & 5.4 \\
$\delta_{3}^{v}$ & 6.6 & 5.6 & 6.0 & $\ldots$ \\
$\delta_{3}^{\theta}$ & 3.9 & 3.8 & 3.8 & $\cdots$ \\
\hline \hline
\end{tabular}

close agreement with the numerical work of Ref. 9. Figure 1 also exhibits an inertial range that is better defined than in Fig. 1 of Ref. 15. We also remark that the width of the scaling region in Fig. 1 is less Reynolds number dependent than the scaling range of the traditional structure functions (not shown). The latter is also notably larger than the former. Lastly, at the largest scales, the inverse structure functions plateau, as also observed in Ref. 9. Similar results (not shown) were obtained for the third-order inverse structure functions. $^{26}$

Inverse structure functions for the temperature field are shown in Fig. 3. Comparing them with the previous results (Figs. 1 and 2), we note that the values of $\delta_{1}^{\theta}, \delta_{2}^{\theta}$, and $\delta_{3}^{\theta}$ are significantly lower than the corresponding values for the velocity field - their values are approximately 1.5, 2.7, and 3.8, respectively. Consequently, the shape of the inverse structure functions appear more "linear," due to the smaller difference between the small-scale and inertial-range scaling exponents. The smaller values of $\delta_{n}^{\theta}$ with respect to $\delta_{n}^{u}$ and $\delta_{n}^{v}$ are related to the intermittency of the passive scalar field, which is more intense than that of the velocity field-see Ref. 27. As for the Reynolds number dependence of the scaling exponents, there appears to be a small tendency towards lower values as the Reynolds number increases.

Figure 4 shows PDFs of $t(|\delta \theta|)$ for small and large values of $\Delta \theta$. It was noted in Ref. 9 that the PDFs of $t\left(\left|\delta u_{i}\right|\right)$ do not tend to Gaussian distributions for large scales as do the PDFs of $\Delta u_{i}$. The same holds for the PDFs of $t(|\delta \theta|)$, which are approximately log-normal. (The deviation from log-normality increases for larger $|\Delta \theta|$.)

Lastly, we point out that "signed" inverse structure functions were also studied (i.e., structure functions conditioned on increments of a given sign, as opposed to ones conditioned on the absolute value of the increment). Though the signed inverse structure functions possessed a different shape
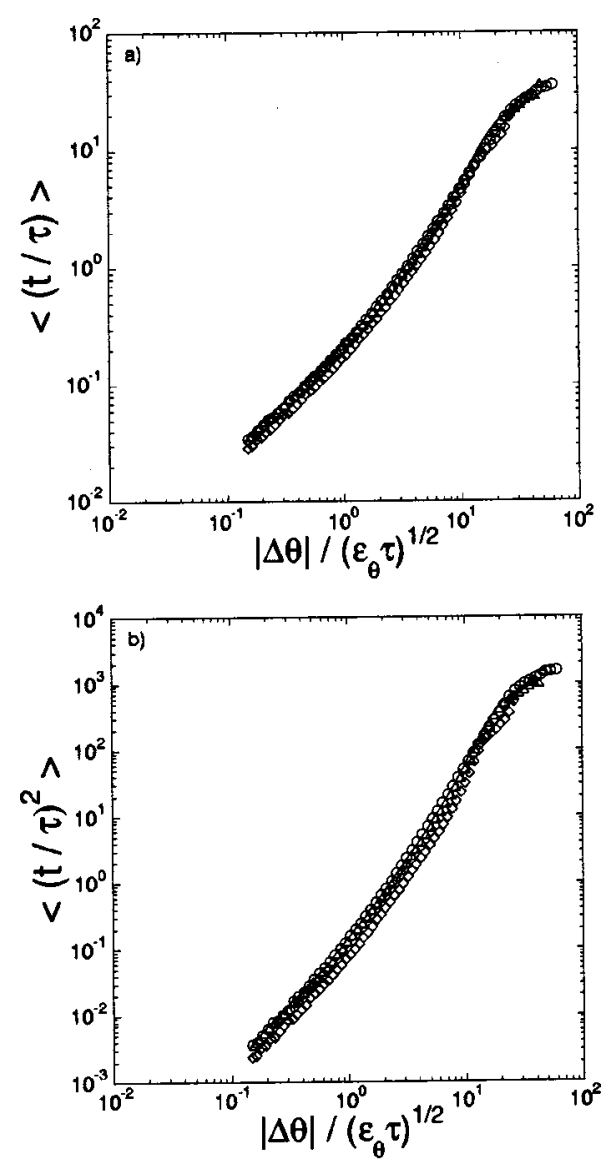

FIG. 3. Inverse structure functions of $\theta$ of the (a) first order and (b) second order. $R_{\lambda}=140(\diamond), R_{\lambda}=306(\triangle), R_{\lambda}=582(\bigcirc)$.

and, therefore, different inertial range slope, the same trends as discussed above were observed. (At small scales, signed inverse structure functions asymptote to a constant value that is equal to the Kolmogorov time scale for the first order. This behavior is attributed to the fact that the average minimum time for the velocity field to exhibit a velocity difference of a given sign is bounded on the lower end by the average time taken for a velocity difference of initially opposite sign to return to zero and become of the desired sign.) Differences between signed inverse structure functions based on positive and negative increments are possible (particularly for $\Delta u$, which is asymmetric). No measurable differences, however, were observed.

In conclusion, results of inverse structure functions of a passive scalar field (temperature) were presented over the range $140 \leqslant R_{\lambda} \leqslant 582$. Their scaling exponents are significantly lower than the corresponding ones of the velocity field - an indication of the stronger internal intermittency in passive scalar fields. The PDFs of $t$ for specified values of $|\Delta \theta|$ were shown to be non-Gaussian at all scales. In addition, inverse structure functions of the longitudinal and transverse velocity fields in grid-generated turbulence were presented. Both were quite similar with inertial-range scaling exponents exhibiting a slight tendency towards smaller values as the Reynolds number is increased. The scaling exponents of both the longitudinal and transverse velocity fields 

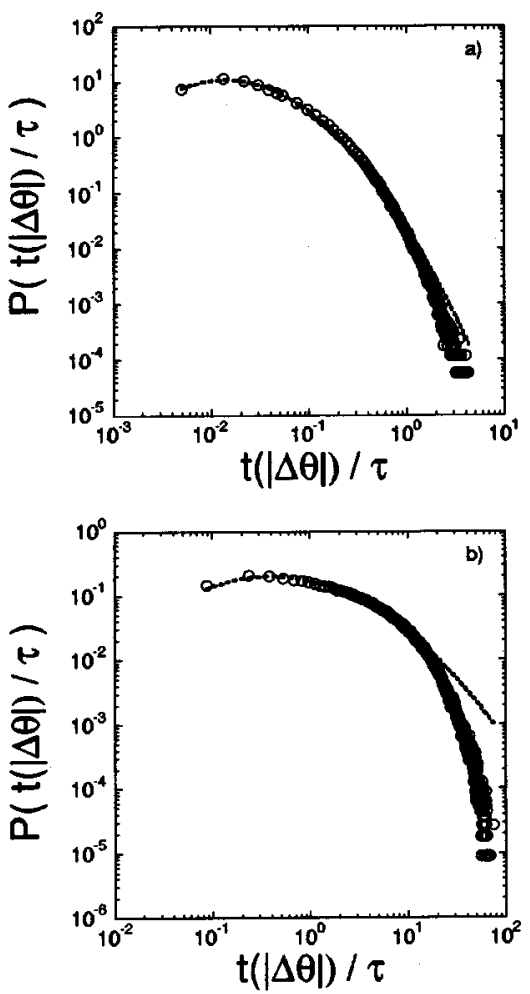

FIG. 4. PDFs of "exit times," $t(|\Delta \theta|)$, for a specified (a) small temperature difference $\left(|\Delta \theta| /\left(\epsilon_{\theta} \tau\right)^{1 / 2}=0.59\right)$ and (b) large temperature difference $\left(|\Delta \theta| /\left(\epsilon_{\theta} \tau\right)^{1 / 2}=11.8\right) . R_{\lambda}=582$. The dashed lines are the best-fit lognormal distributions.

agree with the previous numerical work ${ }^{9}$ and exhibit better defined scaling ranges than observed in Ref. 15.

The authors thank Professor Z. Warhaft for his help with this work. Beneficial comments were also provided by a reviewer. Support was graciously provided by the Natural Sciences and Engineering Research Council of Canada. The measurements were made at Cornell University by means of support from the United States Department of Energy (Basic Energy Sciences).

${ }^{1}$ A. N. Kolmorogov, "The local structure of turbulence in incompressible viscous fluid for very large Reynolds numbers," Dokl. Akad. Nauk SSSR 30, 301 (1941)

${ }^{2}$ A. N. Kolmorogov, "Dissipation of energy in locally isotropic turbulence," Dokl. Akad. Nauk SSSR 32, 16 (1941).

${ }^{3}$ A. M. Oboukhov, "Some specific features of atmospheric turbulence," J. Fluid Mech. 13, 77 (1962).

${ }^{4}$ A. N. Kolmorogov, "A refinement of previous hypotheses concerning the local structure of turbulence in a viscous incompressible fluid at very high Reynolds numbers," J. Fluid Mech. 13, 82 (1962).

${ }^{5}$ F. Anselmet, Y. Gagne, E. J. Hopfinger, and R. A. Antonia, "High-order velocity structure functions in turbulent shear flows," J. Fluid Mech. 140, 63 (1984).

${ }^{6} \mathrm{U}$. Frisch, Turbulence: The Legacy of A. N. Kolmogorov (Cambridge University Press, Cambridge, 1995).

${ }^{7}$ A. Arneodo, C. Baudet, F. Belin, R. Benzi, B. Castaing, B. Chabaud, R.
Chavarria, S. Ciliberto, R. Camussi, F. Chillà, B. Dubrulle, Y. Gagne, B. Hebral, J. Herweiger, M. Marchand, J. Maurer, J. F. Muzy, A. Naert, A. Noullez, J. Peinke, F. Roux, P. Tabeling, W. van de Water, and H. Willaime, "Structure functions in turbulence, in various flow configurations, at Reynolds number between 30 and 5000, using extended self-similarity," Europhys. Lett. 34, 411 (1996).

${ }^{8}$ K. R. Sreenivasan and R. A. Antonia, "The phenomenology of small-scale turbulence,” Annu. Rev. Fluid Mech. 29, 435 (1997).

${ }^{9}$ M. H. Jensen, "Multiscaling and structure functions in turbulence: An alternative approach," Phys. Rev. Lett. 83, 76 (1999).

${ }^{10}$ Jensen used the term "distance structure functions." We prefer "inverse structure functions" (which is used in some of the subsequent literature) because structure functions can be functions of space or time. Note that term "exit time" is also used.

${ }^{11}$ E. B. Gledzer, "System of hydrodynamic type admitting two quadratic integrals of motion," Sov. Phys. Dokl. 18, 216 (1973).

${ }^{12} \mathrm{M}$. Yamada and K. Ohkitani, "Lyapunov spectrum of a chaotic model of three-dimensional turbulence," J. Phys. Soc. Jpn. 56, 4210 (1987).

${ }^{13} \mathrm{M}$. Yamada and K. Ohkitani, "The inertial subrange and non-positive Lyapunov exponents in fully-developed turbulence," Prog. Theor. Phys. 79, 1265 (1988).

${ }^{14} \mathrm{U}$. Frisch and M. Vergassola, "A prediction of the multifractal model: The intermediate dissipation range," Europhys. Lett. 14, 439 (1991).

${ }^{15}$ L. Biferale, M. Cencini, D. Vergni, and A. Vulpiani, "Exit time of turbulent signals: A way to detect the intermediate dissipative range," Phys. Rev. E 60, R6295 (1999).

${ }^{16}$ M. Abel, L. Biferale, M. Cencini, M. Falcioni, D. Vergni, and A. Vulpiani, "Exit-times and epsilon-entropy for dynamical systems, stochastic processes, and turbulence," Physica D 147, 12 (2000).

${ }^{17}$ M. Abel, M. Cencini, M. Falcioni, D. Vergni, A. Vulpiani, and L. Biferale, "On the exit-times approach for epsilon-entropy and turbulent signals," Physica A 280, 49 (2000).

${ }^{18}$ L. Biferale, M. Cencini, A. Lanotte, D. Vergni, and A. Vulpiani, "Inverse statistics of smooth signals: The case of two dimensional turbulence," Phys. Rev. Lett. 87, 124501 (2001).

${ }^{19}$ L. Biferale, M. Cencini, A. S. Lanotte, and D. Vergni, "Inverse velocity statistics in two-dimensional turbulence," Phys. Fluids 15, 1012 (2003).

${ }^{20} \mathrm{H}$. Makita, "Realization of a large-scale turbulence field in a small wind tunnel," Fluid Dyn. Res. 8, 53 (1991).

${ }^{21}$ L. Mydlarski and Z. Warhaft, "On the onset of high Reynolds number grid generated wind tunnel turbulence," J. Fluid Mech. 320, 331 (1996).

${ }^{22}$ An active grid consists of grid bars to which triangular agitator wings are attached. The bars are rotated in different directions at different speeds by means of stepper motors located on the outside of the grid. The result is nearly isotropic grid turbulence with a notably larger turbulence intensity and turbulent Reynolds number.

${ }^{23} \mathrm{~L}$. Mydlarski and Z. Warhaft, "Passive scalar statistics in high-Pécletnumber grid turbulence," J. Fluid Mech. 358, 135 (1998).

${ }^{24}$ J. H. Lienhard, Ph.D. dissertation, University of California at San Diego (1988).

${ }^{25}$ The effects of different methods for calculating the inverse structure functions were studied. Forward lags, backward lags, minimum lags and the average of the forward and backward lags were investigated to determine their effect on $t\left(\left|\Delta u_{i}\right|\right)$. The differences between forward, backward, and average lag calculations were negligible. As would be expected, the difference between the forward and minimum lag calculations was that the value of the minimum-lag inverse structure function was smaller in magnitude than that corresponding to a forward lag. However, the difference in their scaling was minimal. Consequently, forward lags are used in the present work.

${ }^{26}$ Calculation of the PDFs of separations for various values of velocity or temperature difference showed that moments above the third order were not converged. Inverse structure functions converge more slowly than traditional ones.

${ }^{27} \mathrm{Z}$. Warhaft, "Passive scalars in turbulent flows," Annu. Rev. Fluid Mech. 32, 203 (2000). 